\title{
Validação da bateria "Beck" de testes de habilidades para atletas brasileiros de "rugby" em cadeira de rodas
}

CDD. 20.ed. 796.019

796.031

796.333

\author{
J osé Irineu GORLA* \\ Anselmo de Athayde COSTA E SILVA* \\ Leonarto Trevizan COSTA* \\ Luis Felipe Castelli Correia de CAMPOS*
}

\begin{abstract}
Resumo
0 "Rugby" em Cadeira de Rodas é uma modalidade praticada por sujeitos com tetraplegia ou tetraequivalência, desde 2005 no Brasil, onde existem aproximadamente 10 equipes em atividade. A avaliação motora é um dos meios de controle do treinamento que possibilita conquistar avanços no planejamento. A bateria "Beck" de testes de habilidades (manejo de bola, precisão de passes, bloqueio, velocidade e passes de longa distância) para atletas da modalidade foi desenvolvida nos EUA por YILLA e SHERRILL (1998). 0 objetivo deste estudo foi analisar os critérios de autenticidade científica da bateria para a população de atletas brasileiros. Os resultados de duas avaliações com a bateria (teste e re-teste) realizadas com 11 atletas (média de idade 27,59 \pm 5,37 anos, tempo de lesão 8,19 $\pm 3,83$ anos), foram registrados por três avaliadores, o que possibilitou a análise de objetividade, fidedignidade e consistência interna. Foram utilizadas as ferramentas ANOVA para análise de objetividade e, coeficiente de correlação intraclasse e teste $t$ para análise de fidedignidade e consistência. Não foram encontradas diferenças entre os resultados dos diferentes avaliadores (valores de $p$ variando de 0,45 a 1,00) e os valores de correlação intraclasse encontrados (variação de $r$ de 0,78 a 0,99) permitem concluir que a bateria é consistente e fidedigna. Os resultados encontrados neste estudo são evidências de que os critérios de autenticidade científica para a bateria são satisfeitos e a bateria pode ser considerada um instrumento válido para avaliação motora de atletas brasileiros de "rugby" em cadeira de rodas.
\end{abstract}

UnITERmos: Avaliação; Esporte adaptado; Deficiência física.

\section{Introdução}

O "Rugby" em Cadeira de Rodas (RCR) é um esporte Paraolímpico que foi criado no Canadá, nos anos 70, como uma alternativa de prática para pessoas com tetraplegia. Inicialmente o esporte era uma alternativa para aqueles atletas com limitações de força nos membros superiores que não tinham espaço de prática no Basquete em Cadeira de Rodas. O RCR é jogado por dois times de quatro jogadores cada (e quatro reservas adicionais) em uma quadra com as mesmas dimensões da quadra de basquetebol ( $28 \times 15$ metros). Os gols são anotados quando os atletas ultrapassam o gol na linha de fundo adversária carregando a bola (espaço de oito metros, demarcados no centro das linhas de fundo por dois cones).
Atualmente segundo dados da AsSOCIAÇÃo BraSILEIra de Rugby em Cadeira de Rodas (ABRC, 2019) o "rugby" é presente em 32 países. No Brasil a modalidade é praticada desde 2005 (SANT'ANA, 2011) e no momento da escrita deste estudo existiam 10 equipes praticantes da modalidade participando ativamente de competiçōes.

Assim como no esporte convencional a Avaliação Motora desempenha um papel fundamental no esporte paraolímpico. Sua importância é determinada pela capacidade de fornecer subsídios para uma intervenção consciente, e também: a) permite estabelecer metas em relação às capacidades físicas dos sujeitos, para o programa de treinamento; b) permite o diagnóstico do nível de desempenho do atleta; c) 
permite estabelecer parâmetros de comparação dentro da modalidade o que é útil para o planejamento e; d) fornece subsídios para a ação dos Classificadores.

Sendo o "Rugby" um esporte de prática recente no Brasil são necessários estudos no sentido de prover instrumentos de avaliação específicos que venham a ser úteis para os treinadores da modalidade. YILla e SHERRILL (1998) validaram uma bateria especifica de testes de habilidades para o "Rugby" em Cadeira de Rodas: "Beck Battery of Quad Rugby Skills Tests". Os testes propostos estão centrados na avaliação das habilidades de manejo de bola (fidedignidade, $R=0,97$ ), bloqueio (fidedignidade, $\mathrm{R}=0,98$ ), deslocamento em

\section{Métodos}

Este estudo é caracterizado de acordo com THOmas, Nelson e Silverman (2007) como pesquisa descritiva, de caráter correlacional e apresenta um delineamento transversal. Envolveu variáveis que permitem avaliar o desempenho de sujeitos praticantes de "rugby" em cadeira de rodas em habilidades como: manejo de bola (pontuação obtida durante $30 \mathrm{~s}$ ), precisão de passes (pontos obtidos), bloqueio (tempo do teste em segundos), velocidade velocidade (fidedignidade, $\mathrm{R}=0,99$ ), precisão de passes (fidedignidade, $\mathrm{R}=0,94$ ) e desempenho dos passes de longa distância (fidedignidade, $\mathrm{R}=0,97$ ), todas determinantes da modalidade.

Acredita-se que esta bateria seja um instrumento importante para a avaliação dos atletas de "rugby" e, assim justifica-se o presente estudo cujo objetivo principal foi o de analisar os critérios de autenticidade científica da bateria "Beck" para avaliação de atletas brasileiros da modalidade. A relevância do presente estudo deve-se à sua capacidade de fornecer um instrumento válido para uma avaliação específica da modalidade e assim contribuir para o controle do treinamento na área.

(tempo em segundos do deslocamento no percurso de 20 metros) e, passes de longa distância (pontos obtidos).

Em relação ao desenho, o estudo foi composto por teste e re-teste (através deste procedimento se avaliou fidedignidade e consistência interna), nos quais os sujeitos foram avaliados por três avaliadores com o objetivo de analisar a objetividade dos testes, conforme descrito na FIGURA 1 a seguir:

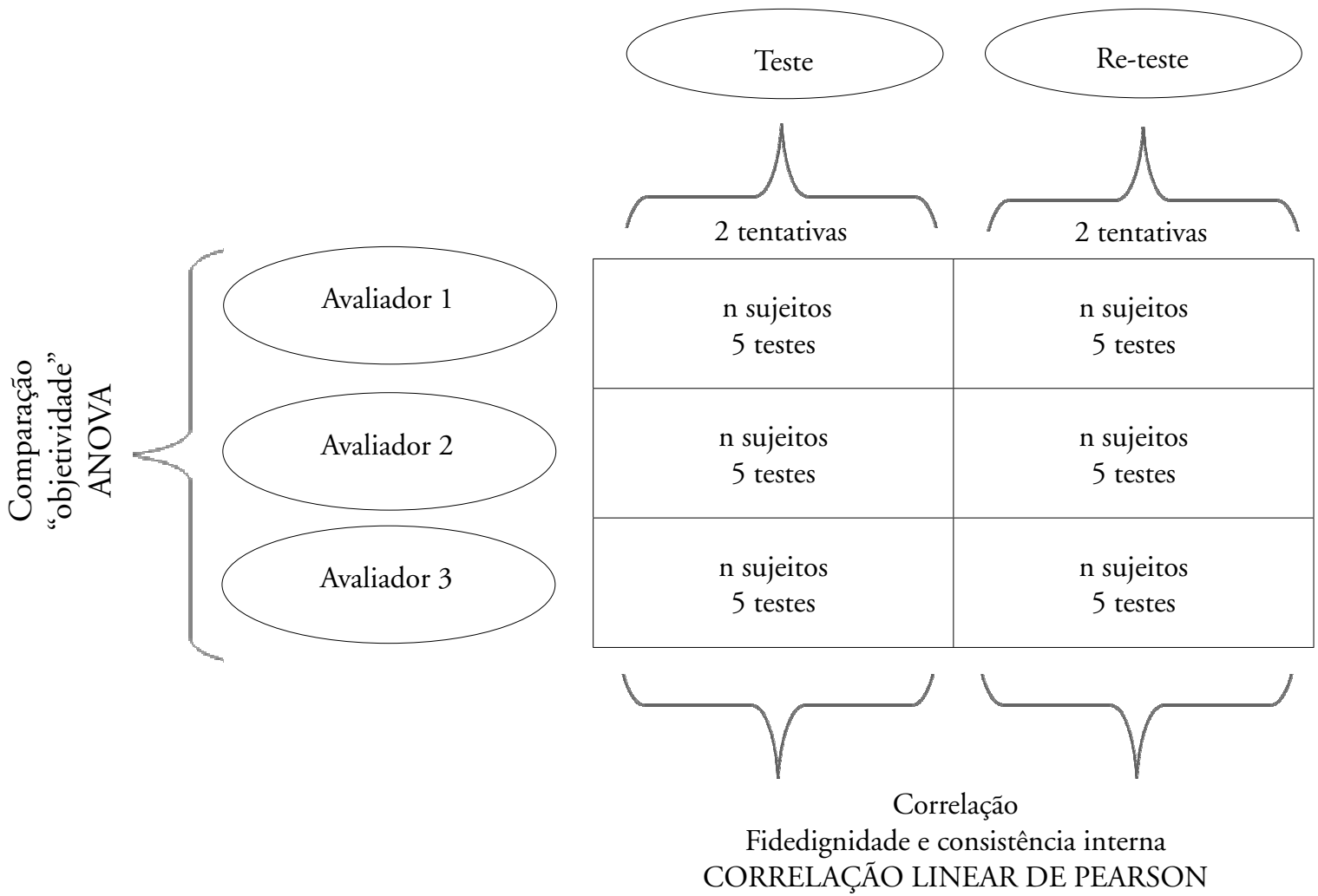

FIGURA 1 - Desenho do estudo. 
Assumiu-se que a bateria possuía validade de conteúdo, por conta de a mesma já ter sido validada nos Estados Unidos para avaliação de atletas da modalidade. A objetividade pode ser entendida como o grau em que avaliadores diferentes são capazes de encontrar valores consistentes através de um mesmo teste. A correlação entre as diferentes medidas (teste e re-teste) realizadas pelos mesmos avaliadores foi utilizada para estabelecer a fidedignidade dos testes que representa o grau em que um mesmo avaliador obtém medidas consistentes. A consistência interna foi analisada através da correlação entre os resultados das duas tentativas (i.e. duas tentativas no teste e, duas no re-teste).

\section{Amostra}

A população deste estudo foi composta por atletas de "rugby" em cadeira de rodas com lesão medular ou alguma deficiência que cause comprometimento motor equivalente à tetraplegia (em outras palavras, comprometimento dos quatro membros). No momento da coleta de dados existiam 10 equipes praticando a modalidade no Brasil com um número estimado em aproximadamente 100 atletas. A amostra deste estudo foi composta por 11 atletas do sexo masculino, média de idade 27,59 $( \pm 5,37$ anos, mín.: 21, máx. 36 anos), tempo de lesão 8,19 ( \pm 3,83 anos, mín.: 3,8, máx. 16 anos), praticantes da modalidade há no mínimo um ano, e que participaram de pelo menos uma competição nacional.

Quanto ao nível de lesão medular, as lesōes variaram dos segmentos cervicais C5-C6 ao segmento torácico T2. Em relação à classificação funcional os participantes foram classificados variando de 0,5 a 3,0 (um atleta 0,5; três atletas 1,0 ; um atleta 2,0; cinco atletas 2,50 e um atleta 3,0 ). Todos os participantes assinaram o termo de consentimento livre e esclarecido para participação na pesquisa, que esteve em conformidade com os parâmetros de ética em pesquisa da resolução de ética em pesquisa envolvendo seres humanos no. 196/96 e foi aprovado pelo Comitê de Ética de Pesquisa em estudos com seres humanos da Faculdade de Medicina da UNICAMP sob o n. de protocolo 229/2009.

Os atletas participantes do estudo integravam a equipe campeã nacional nos anos de 2009 e 2010, sendo que sete dos participantes eram integrantes da seleção brasileira. A equipe avaliada venceu todos os seus confrontos no campeonato brasileiro de 2010 por uma diferença superior a de 30 pontos. Este fator permitiu que o estudo fosse desenvolvido com uma amostra reduzida, pois devido à diferença de potencial entre as equipes brasileiras, optou-se por realizar a avaliação apenas com atletas que participassem de um programa de treinamento da modalidade para garantir que a falta de treinamento não fosse um fator limitante do estudo. Pela mesma razão optou-se por avaliar atletas com experiência de no mínimo uma competição.

\section{Procedimentos}

Os participantes do estudo foram instruídos para não consumir álcool e nem fazer exercícios no período de 16 horas antes das coletas de dados conforme o procedimento adotado por MoJTAHEDI, VALENTINE e Evans (2009). A mesma foi realizada no ambiente de treinamento dos atletas, sendo que nos dias das avaliações os atletas não realizaram os treinos. As coletas foram separadas entre si por um intervalo de 48 horas ou dois dias. Para a realização dos testes, cada sujeito avaliado utilizava a sua cadeira de jogo além dos acessórios habituais, tais como: ataduras, luvas e faixas. Cada participante dispunha de uma tentativa ensaio em cada teste para familiarização com o protocolo.

A avaliação foi conduzida sempre pelo mesmo avaliador, contudo, dois outros avaliadores realizaram a tomada dos resultados de forma a permitir a avaliação da objetividade dos testes. A primeira avaliação foi filmada com o objetivo de construir um vídeo instrucional de divulgação da bateria de testes. O vídeo será posteriormente enviado às demais equipes, para que a coleta de dados possa ser realizada com uma amostra maior e para que todas as equipes tenham acesso aos procedimentos de avaliação.

Da primeira avaliação (teste), os dois avaliadores participantes realizaram a tomada dos resultados através de análise do conteúdo das filmagens realizadas na primeira avaliação. No segundo momento (re-teste) os avaliadores participaram da avaliação em conjunto com o avaliador principal e fizeram a tomada das medidas simultaneamente. Os resultados computados pelos três avaliadores foram tabulados e foi calculada a estatística descritiva geral e também por classe, sendo que os atletas foram divididos entre classe funcional baixa $<2,0$ e classe funcional alta $>2,0$. Este procedimento cumpre o objetivo de estabelecer valores referenciais para os atletas da modalidade.

\section{Protocolo - Bateria “Beck”}

Os sujeitos foram submetidos a um protocolo de avaliação motora em teste e re-teste através da bateria "Beck" de habilidades para "rugby" em cadeira de rodas. A bateria consiste em cinco testes que foram criados 
especificamente para avaliar as habilidades do "rugby" em cadeira de rodas e, cada teste é composto por duas tentativas sendo que o melhor resultado é computado para avaliação (detalhamento no ANEXO I):

1) Teste de manejo de bola (MAN) - neste teste $o$ atleta deve percorrer um percurso delimitado por cones durante o período de 30 segundos conduzindo a bola de acordo com as regras da modalidade, ou seja, a cada 10 segundos deve ser executado um drible e, no restante do tempo a bola pode estar no colo. O percurso é demarcado em um espaço de $10 \mathrm{~m}^{2}$. Os cones do percurso forma "portas" e, ao dar a volta em cada porta o atleta marca um ponto. O número de pontos marcados ao final do teste é o resultado. O percurso é apresentado no ANEXO I com maiores detalhes.

2) Teste de precisão de passes (PREC) - neste teste é desenhado um alvo na parede, cuja distância do sujeito avaliado é de cinco metros. A cada metro do sujeito à parede é passada uma linha, separando espaços cujos valores vão de um ponto na primeira parte à frente do avaliado ao centro do alvo que é de 10 pontos. A altura do alvo em relação ao chão é de $1,15 \mathrm{~m}$. O teste consiste em realizar passes até o alvo para quantificar a pontuação e o passe que $\mathrm{o}$ atleta utiliza em quadra deve ser utilizado no teste. Em cada tentativa do teste (duas ao todo) são três execuções. A soma dos pontos obtidos em cada tentativa é o resultado do teste.

3) Teste de desempenho de bloqueio (BLOQ) - este teste mensura a habilidade de bloqueio que é determinante para o desempenho do atleta nas partidas de "rugby" em cadeira de rodas. $\mathrm{O}$ teste consiste em um percurso de aproximadamente 24 metros, com seis cones colocados em linha reta, e com a distância de três metros entre cada um deles, e mais uma distância de 1,5 entre o início do percurso e o primeiro cone, bem como do último cone e término do percurso. Levando em consideração que a base dos cones pode variar de tamanho estima-se em 24 metros a área de execução. Contudo para o presente estudo a distância foi de 22,8 metros por conta da largura dos cones. $\mathrm{O}$ atleta deve simular a ação do bloqueio em cada um dos cones. $\mathrm{O}$ resultado é o tempo em segundos da ação dos atletas.

4) Teste de velocidade 20 metros (VEL) - este teste consiste em mensurar a habilidade de deslocamento em velocidade. Utiliza-se um percurso de 20 metros. $\mathrm{O}$ avaliador posiciona-se ao final do percurso e um auxiliar fica posicionado ao início para dar o sinal que corresponde à saída do atleta. $\mathrm{O}$ ponto de referência para o teste é a passagem do par de rodas dianteiras da cadeira, logo, quando o par de rodas dianteiras passa a linha o auxiliar que está com o braço elevado, abaixa-o para sinalizar ao avaliador que inicia a contagem do tempo com o cronômetro. $\mathrm{O}$ atleta desloca-se então em velocidade e quando passa pela linha final, o segundo avaliador para o cronômetro. O tempo em segundos é computado e são duas tentativas válidas no teste.

5) Teste de passes de longa distância (PASSE) - este é o teste que visa mensurar a habilidade dos atletas em efetuar os passes de longa distância. $\mathrm{O}$ participante posiciona-se à linha de fundo da quadra e, deve realizar o passe de longa distância com a maior força possível, utilizando o passe realizado durante a situação de jogo. Lateralmente à quadra são demarcados espaços de dois em dois metros com cones que são as zonas de referencia para a pontuação do teste. As zonas são enumeradas com escala de razão (1) a partir do número um. A pontuação da zona em que a bola toca ao chão é o resultado da execução. São três execuções em cada uma das duas tentativas. A soma em cada tentativa é o resultado do teste.

\section{Materiais}

Para a coleta de dados foram utilizados os seguintes materiais: um cronômetro digital da marca Cássio ${ }^{\circledR}$, dois cronômetros digitais da marca Technos ${ }^{\circledR}$, todos com precisão de centésimos de segundos, uma filmadora Sony HandyCam ${ }^{\circledR}$ HDR - XR 100, 4.0 megapixels, fitas adesivas e cones de plástico com base de $0,30 \mathrm{~m}$ de lado, além de uma trena com precisão de centímetros, para demarcação dos percursos e alvo.

\section{Análise estatística}

Quanto à análise estatística, utilizou-se o pacote estatístico SPSS $10.0{ }^{\circledR}$ para Windows ${ }^{\circledR}$. Com a estatística descritiva foram apresentados os dados através de média, desvio padrão, erro padrão da média, valores mínimo e máximo, intervalo de confiança da média a 95\%, Percentis 25, 50 e 75, além da amplitude interquartil. A análise da normalidade dos dados foi feita através do teste de Shapiro-Wilk. Nos casos em que os dados apresentaram normalidade utilizou-se a Análise de Variância de um fator (ANOVA one-way) para a análise da objetividade. A análise da fidedignidade foi realizada através do teste " $\mathrm{t}$ " de Student para amostras pareadas e do coeficiente de correlação intra-classe. $\mathrm{O}$ mesmo procedimento de correlação foi realizado para avaliar a consistência interna dos testes. Para a comparação dos valores entre jogadores de classe funcional alta e baixa, utilizou-se o teste de Mann Whitney porque a distribuição dos dados não foi considerada normal. O nível de significância adotado no estudo foi de 5\%. 


\section{Resultados}

Cada teste foi realizado duas vezes com o mesmo atleta e no caso dos testes quantificados em tempo (segundos) o melhor tempo foi utilizado para as análises. Para os testes em que o resultado é expresso por uma pontuação específica, i.e., precisão de passes e passes de longa distância, foi realizada uma soma dos resultados para cada uma das duas tentativas. Em cada avaliação os atletas executavam duas tentativas de cada teste e, com isso, para as análises foram dispostos conjuntos de quatro variáveis para cada teste. As variáveis foram denominadas "a" e "b" para primeira avaliação, e, "c" e "d" para segunda avaliação, (por exemplo: "Man_a"; primeira tentativa do teste de manejo de bola na primeira avaliação).

$\mathrm{Na}$ TABELA 1 são apresentados os resultados da estatística descritiva para os testes de manejo de bola, precisão de passes e arremessos e desempenho de bloqueio. Na TABELA 2 são apresentados os resultados dos testes de velocidade e passe de longa distância. Assim como na TABELA 1, é apresentada a estatística descritiva para as quatro tentativas de cada teste. São apresentados também os valores descritivos de cada avaliador em cada uma das tentativas para permitir a comparação e subsequente análise do critério de objetividade. Para a análise do critério de fidedignidade foi realizada a comparação entre as quatro medidas realizadas por cada avaliador. Portanto nas TABELAS 1 e 2, as duplas de variáveis que apresentaram diferenças estatisticamente significativas foram identificadas com um algarismo sobrescrito (por exemplo: 6,45 ${ }^{\mathrm{x}}$ e $6,91^{\mathrm{x}}$ ).

Não foram encontradas diferenças significativas entre os resultados dos três avaliadores participantes do estudo. Com isso o critério de objetividade da bateria é satisfeito, pois diferentes avaliadores são capazes de obter medidas consistentes dos resultados dos testes. Quanto aos critérios de fidedignidade e consistência interna foram encontradas algumas diferenças significativas para alguns dos testes, o que pode ser indicativo de baixa fidedignidade. Para o avaliador 1 foram encontradas diferenças significativas nos testes de desempenho de bloqueio, para as seguintes variáveis: $\mathrm{BLOQ} A \mathrm{~A}$ x BLOQ_B $\left({ }^{\mathrm{a}} \mathrm{p}=0,03\right)$, BLOQ_A x BLOQ_C $\left({ }^{b} p=0,03\right)$ e, BLOQ_A $x$ BLOQ_D $\left({ }^{c} p=\right.$ 0,03). PASSE_A $x$ PASSE_B $\left({ }^{\mathrm{d}} \mathrm{p}=0,03\right)$ e PASSE_A $x$ PASSE_C $\left({ }^{e} p=0,05\right)$. Os resultados do segundo avaliador apresentaram diferenças significativas nas variáveis: PREC_B x PREC_C ( $\mathrm{i} \mathrm{p}=0,02), \mathrm{BLOQ} A \mathrm{~A}$ x BLOQ_B (ii $\mathrm{p}=0,02)$ e BLOQ_A x BLOQ_C (iiip $=0,01$ ). Já o terceiro avaliador apresentou diferenças significativas para os testes de manejo de bola: MAN_B x MAN_C $\left({ }^{\mathrm{x}} \mathrm{p}=0,02\right)$, PREC_B x PREC_C ( $\left({ }^{\mathrm{y}} \mathrm{p}=0,03\right)$, BLOQ_B $\times$ BLOQ_C $\left({ }^{\mathrm{z}} \mathrm{p}=\right.$ $0,05)$ e PASSE_A $\times$ PASSE_D $\left({ }^{w} p=0,05\right)$.

TABELA 1 - Resultados dos testes demanejo de bola (pontos), precisão de passes (pontos) ebloqueio (segundos)..

\begin{tabular}{|c|c|c|c|c|c|c|c|c|c|c|c|c|c|c|}
\hline \multirow[b]{2}{*}{ VAR } & \multirow[b]{2}{*}{ AV } & \multirow[b]{2}{*}{$\mathbf{M}$} & \multirow[b]{2}{*}{ DP } & \multirow[b]{2}{*}{ EP } & \multicolumn{2}{|c|}{ IC95\% } & \multirow[b]{2}{*}{ MIN } & \multirow[b]{2}{*}{ MAX } & \multirow[b]{2}{*}{ P25 } & \multirow[b]{2}{*}{ P50 } & \multirow[b]{2}{*}{ P75 } & \multirow[b]{2}{*}{ AIQ } & \multirow[b]{2}{*}{ ANOVA } & \multirow{3}{*}{$\begin{array}{l}\text { Man: teste de manejo } \\
\text { de bola; }\end{array}$} \\
\hline & & & & & INF & SUP & & & & & & & & \\
\hline \multirow{3}{*}{ MAN_A } & 1 & 6,82 & 1,94 & 0,58 & 5,51 & 8,12 & 4,00 & 10,00 & 5,50 & 7,00 & 7,50 & 3,00 & $F-0,17$ & \\
\hline & 2 & 6,45 & 2,25 & 0,68 & 4,94 & 7,97 & 4,00 & 10,00 & 4,50 & 7,00 & 7,50 & 4,00 & \multirow[t]{2}{*}{$P-0,85$} & $\begin{array}{l}\text { Prec: teste de precisão } \\
\text { de passes; }\end{array}$ \\
\hline & 3 & 6,55 & 2,34 & 0,70 & 4,97 & 8,12 & 4,00 & 10,00 & 4,50 & 7,00 & 8,00 & 5,00 & & Bloq: teste de desempe- \\
\hline \multirow{3}{*}{ MAN_B } & 1 & 6,82 & 1,89 & 0,57 & 5,55 & 8,09 & 4,00 & 10,00 & 5,50 & 7,00 & 8,00 & 4,00 & \multirow{3}{*}{$\begin{array}{l}F-0,28 \\
P-0,76\end{array}$} & $\begin{array}{l}\text { nho de bloqueio; } \\
\text { AV: Avaliador; }\end{array}$ \\
\hline & 2 & 6,55 & 2,21 & 0,66 & 5,06 & 8,03 & 3,00 & 10,00 & 5,00 & 7,00 & 7,50 & 3,00 & & Nível de significância \\
\hline & 3 & $6,45^{x}$ & 2,07 & 0,62 & 5,06 & 7,84 & 3,00 & 10,00 & 5,00 & 7,00 & 7,50 & 3,00 & & $a: p=0,032$ \\
\hline \multirow{3}{*}{ MAN_C } & 1 & 6,91 & 1,97 & 0,59 & 5,58 & 8,23 & 4,00 & 10,00 & 6,00 & 7,00 & 8,50 & 3,00 & \multirow{3}{*}{$\begin{array}{l}F-0,06 \\
P-0,94\end{array}$} & $\begin{array}{l}b: p=0,035 \\
c: p=0,030\end{array}$ \\
\hline & 2 & 6,82 & 1,99 & 0,60 & 5,48 & 8,15 & 4,00 & 10,00 & 5,00 & 7,00 & 8,50 & 4,00 & & i: $p=0,018$ \\
\hline & 3 & $6,91^{x}$ & 2,16 & 0,65 & 5,45 & 8,36 & 4,00 & 11,00 & 5,00 & 7,00 & 8,50 & 4,00 & & $\begin{array}{l}\text { ii: } p=0,02 \\
\text { iii: } p=0,01\end{array}$ \\
\hline \multirow{3}{*}{ MAN_D } & 1 & 6,73 & 2,15 & 0,65 & 5,28 & 8,17 & 3,00 & 10,00 & 5,00 & 7,00 & 8,50 & 4,00 & \multirow{3}{*}{$\begin{array}{l}F-0,01 \\
P-0,99\end{array}$} & $x: p=0,02$ \\
\hline & 2 & 6,73 & 2,15 & 0,65 & 5,28 & 8,17 & 3,00 & 10,00 & 5,00 & 7,00 & 8,50 & 4,00 & & $\begin{array}{l}y: p=0,03 \\
z: p=0,05\end{array}$ \\
\hline & 3 & 6,64 & 2,11 & 0,64 & 5,22 & 8,05 & 3,00 & 10,00 & 5,00 & 7,00 & 8,00 & 4,00 & & \\
\hline
\end{tabular}

Continua 
TABELA 1 - Resultados dos testes de manejo de bola (pontos), precisão de passes (pontos) ebloqueio (segundos) - Continuação).

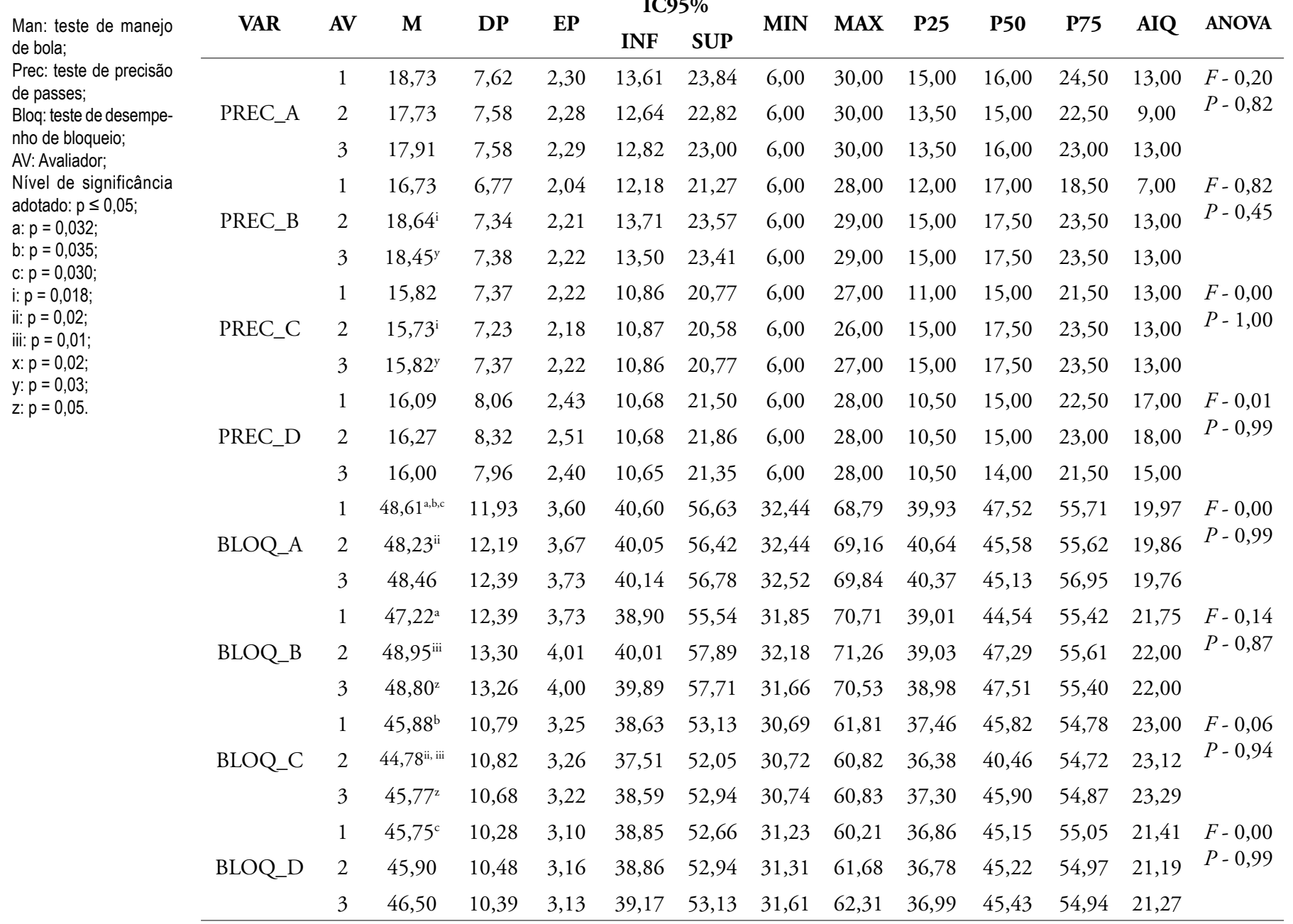

TABELA 2 - Resultados dos testes de velocidade 20 metros (segundos) e passes de longa distância (pontos).

Vel: teste de velocidade 20 metros;

Passe: teste de passes de longa distância;

AV: Avaliador;

Nível de significância

adotado: $p \leq 0,05$;

$\mathrm{d}: \mathrm{p}=0,033$;

e: $p=0,048$;

$w: p=0,05$.

\begin{tabular}{|c|c|c|c|c|c|c|c|c|c|c|c|c|c|}
\hline \multirow{2}{*}{ VAR } & \multirow{2}{*}{ AV } & \multirow{2}{*}{$\mathbf{M}$} & \multirow{2}{*}{ DP } & \multirow{2}{*}{ EP } & \multicolumn{2}{|c|}{ IC95\% } & \multirow{2}{*}{ MIN } & \multirow{2}{*}{ MAX } & \multirow{2}{*}{ P25 } & \multirow{2}{*}{ P50 } & \multirow{2}{*}{ P75 } & \multirow{2}{*}{ AIQ } & \multirow{2}{*}{ ANOVA } \\
\hline & & & & & INF & SUP & & & & & & & \\
\hline \multirow{3}{*}{ VM_A } & 1 & 8,85 & 2,50 & 0,75 & 7,17 & 10,54 & 5,94 & 13,37 & 7,21 & 8,17 & 9,94 & 3,95 & \multirow{3}{*}{$\begin{array}{l}F-0,05 \\
P-0,95\end{array}$} \\
\hline & 2 & 8,70 & 2,42 & 0,73 & 7,08 & 10,33 & 5,90 & 13,63 & 6,93 & 8,02 & 9,60 & 3,33 & \\
\hline & 3 & 8,66 & 2,42 & 0,73 & 7,04 & 10,29 & 6,02 & 13,55 & 6,91 & 7,98 & 9,61 & 3,58 & \\
\hline \multirow{3}{*}{ VM_B } & 1 & 8,81 & 2,40 & 0,72 & 7,19 & 10,42 & 5,66 & 13,43 & 7,39 & 8,08 & 10,09 & 3,40 & \multirow{3}{*}{$\begin{array}{l}F-0,00 \\
P-1,00\end{array}$} \\
\hline & 2 & 8,78 & 2,70 & 0,81 & 6,96 & 10,59 & 5,67 & 13,63 & 7,13 & 7,87 & 9,93 & 3,54 & \\
\hline & 3 & 8,80 & 2,68 & 0,81 & 7,00 & 10,61 & 5,60 & 13,80 & 7,27 & 7,87 & 9,80 & 3,13 & \\
\hline \multirow{3}{*}{ VM_C } & 1 & 8,69 & 2,53 & 0,76 & 6,99 & 10,39 & 5,73 & 13,91 & 6,93 & 7,86 & 9,83 & 3,41 & \multirow{3}{*}{$\begin{array}{l}F-0,01 \\
P-0,99\end{array}$} \\
\hline & 2 & 8,65 & 2,28 & 0,69 & 7,12 & 10,19 & 5,77 & 13,04 & 7,10 & 7,79 & 9,87 & 3,27 & \\
\hline & 3 & 8,77 & 2,35 & 0,71 & 7,19 & 10,35 & 5,92 & 13,28 & 7,01 & 8,10 & 9,97 & 3,47 & \\
\hline \multirow{3}{*}{ VM_D } & 1 & 8,69 & 2,46 & 0,74 & 7,04 & 10,35 & 5,70 & 12,81 & 6,91 & 7,66 & 9,98 & 3,48 & \multirow{3}{*}{$\begin{array}{l}F-0,00 \\
P-1,00\end{array}$} \\
\hline & 2 & 8,66 & 2,45 & 0,74 & 7,02 & 10,31 & 5,76 & 12,96 & 6,96 & 7,63 & 9,93 & 3,61 & \\
\hline & 3 & 8,68 & 2,55 & 0,77 & 6,97 & 10,39 & 5,83 & 12,97 & 6,86 & 7,58 & 9,96 & 3,62 & \\
\hline
\end{tabular}

Continua

478 • Rev. bras. Educ. Fís. Esporte, São Paulo, v.25, n.3, p.473-86, jul./ set. 2011 
TABELA 2 - Resultados dos testes de velocidade 20 metros (segundos) e passes de longa distância (pontos) (Continuação).

\begin{tabular}{|c|c|c|c|c|c|c|c|c|c|c|c|c|c|}
\hline \multirow{2}{*}{ VAR } & \multirow{2}{*}{ AV } & \multirow{2}{*}{$\mathbf{M}$} & \multirow{2}{*}{ DP } & \multirow{2}{*}{ EP } & \multicolumn{2}{|c|}{ IC95\% } & \multirow{2}{*}{ MIN } & \multirow{2}{*}{ MAX } & \multirow{2}{*}{ P25 } & \multirow{2}{*}{ P50 } & \multirow{2}{*}{ P75 } & \multirow{2}{*}{ AIQ } & \multirow{2}{*}{ ANOVA } \\
\hline & & & & & INF & SUP & & & & & & & \\
\hline \multirow{3}{*}{ PASSE_A } & 1 & $11,27^{\mathrm{d}}$ & 5,18 & 1,56 & 7,79 & 14,75 & 3,00 & 21,00 & 8,50 & 10,00 & 14,50 & 7,00 & \multirow{3}{*}{$\begin{array}{l}F-0,37 \\
P-0,96\end{array}$} \\
\hline & 2 & 11,18 & 5,19 & 1,56 & 7,69 & 14,67 & 3,00 & 21,00 & 8,50 & 10,00 & 13,50 & 6,00 & \\
\hline & 3 & $11,09^{\mathrm{w}}$ & 5,22 & 1,57 & 7,58 & 14,60 & 3,00 & 21,00 & 8,50 & 9,00 & 13,50 & 6,00 & \\
\hline \multirow{3}{*}{ PASSE_B } & 1 & $12,18^{\mathrm{d}, \mathrm{e}}$ & 5,21 & 1,57 & 8,68 & 15,68 & 4,00 & 21,00 & 9,00 & 11,00 & 16,00 & 8,00 & \multirow{3}{*}{$\begin{array}{l}F-0,29 \\
P-0,75\end{array}$} \\
\hline & 2 & 11,18 & 5,06 & 1,52 & 7,78 & 14,58 & 4,00 & 20,00 & 8,00 & 9,00 & 15,50 & 10,00 & \\
\hline & 3 & 11,18 & 5,21 & 1,57 & 7,68 & 14,68 & 4,00 & 20,00 & 8,00 & 9,00 & 15,50 & 10,00 & \\
\hline \multirow{3}{*}{ PASSE_C } & 1 & $10,00^{\mathrm{e}}$ & 5,46 & 1,65 & 6,33 & 13,67 & 3,00 & 20,00 & 6,00 & 9,00 & 13,50 & 9,00 & \multirow{3}{*}{$\begin{array}{l}F-0,00 \\
P-1,00\end{array}$} \\
\hline & 2 & 9,91 & 5,48 & 1,65 & 6,22 & 13,59 & 3,00 & 20,00 & 6,00 & 9,00 & 13,50 & 9,00 & \\
\hline & 3 & 9,82 & 5,51 & 1,66 & 6,12 & 13,52 & 3,00 & 20,00 & 6,00 & 8,00 & 13,50 & 9,00 & \\
\hline \multirow{3}{*}{ PASSE_C } & 1 & 9,82 & 5,71 & 1,72 & 5,98 & 13,65 & 3,00 & 21,00 & 6,00 & 9,00 & 12,50 & 7,00 & \multirow{3}{*}{$\begin{array}{l}F-0,01 \\
P-0,98\end{array}$} \\
\hline & 2 & 10,00 & 5,71 & 1,72 & 6,16 & 13,84 & 3,00 & 21,00 & 5,50 & 9,00 & 13,00 & 9,00 & \\
\hline & 3 & $9,82^{\mathrm{w}}$ & 5,83 & 1,76 & 5,90 & 13,74 & 3,00 & 21,00 & 5,50 & 8,00 & 13,00 & 9,00 & \\
\hline
\end{tabular}

Vel: teste de velocidade 20 metros;

Passe: teste de passes de longa distância; AV: Avaliador; Nível de significância adotado: $p \leq 0,05$; $\mathrm{d}: \mathrm{p}=0,033$; e: $p=0,048$ w: $p=0,05$.
Contudo na TABELA 3 é apresentada a matriz de correlação intraclasse para os três avaliadores e todas as variáveis. Esta avaliação permite confirmar que embora sejam apresentadas para as variáveis anteriormente citadas, diferenças estatisticamente significativas entre os pares, o critério de fidedignidade é satisfeito porque, para os pares que apresentaram diferenças significativas, os valores de correlação foram considerados aceitáveis dentro da classificação proposta por TRITSCHLER (2000) $(0,80$ a 0,89 - correlação muito boa e, 0,90 à 0,99 - excelente). Quanto à consistência interna dos testes, as únicas variáveis que apresentaram debilidades foram às correspondentes ao teste de passes de longa distância (Avaliador 1: PASSE_B x PASSE_D - R $=0,76$ e; Avaliador 2: PASSE_B x PASSE_C - R $=0,78)$. Foram encontrados valores de $\mathrm{R}$ de 0,81 a 0,99 para os testes de precisão de passes (para o avaliador 1: A x B, A x C, A x D; Avaliador 2: A x B, A x C; Avaliador 3: A x B, A x D, B x C) e para o teste de passes de longa distância foi encontrada a variação de $\mathrm{R}$ da ordem de 0,76 a 0,99 (Avaliador 1: A x C, B x C, B x D; Avaliador 2: B x C, B x D; Avaliador 3: B x C, B x D, p $\leq 0,05)$. Embora estes valores possam ser analisados como valores fortes de correlação, há indicativos de que a consistência interna do teste apresente problemas.

Na TABELA 4 é apresentada à estatística descritiva para as variáveis do estudo em separado para atletas de classe funcional baixa $(<2,0, \mathrm{n}=6)$ e alta $(>2,0$, $\mathrm{n}=5$ ). Para todas as variáveis os atletas de pontuação alta apresentaram um desempenho superior estatisticamente significativo, na comparação realizada através do teste de Mann Whitney $(\mathrm{p} \leq 0,05)$. 
TABELA 3 - Matriz de correlação das variáveis para cada avaliador.

\begin{tabular}{|c|c|c|c|c|c|c|c|c|c|c|c|c|}
\hline \multirow{2}{*}{ Variável } & \multicolumn{4}{|c|}{ Avaliador 1} & \multicolumn{4}{|c|}{ Avaliador 2} & \multicolumn{4}{|c|}{ Avaliador 3} \\
\hline & A & B & C & D & $\mathbf{A}$ & B & C & D & A & B & $\mathrm{C}$ & D \\
\hline MAN_A & 1,00 & - & - & - & 1,00 & - & - & - & 1,00 & - & - & - \\
\hline MAN_B & 0,95 & 1,00 & - & - & 0,97 & 1,00 & - & - & 0,96 & 1,00 & - & - \\
\hline MAN_C & 0,88 & 0,96 & 1,00 & - & 0,94 & 0,96 & 1,00 & - & 0,94 & 0,97 & 1,00 & \\
\hline MAN_D & 0,90 & 0,97 & 0,96 & 1,00 & 0,94 & 0,96 & 0,97 & 1,00 & 0,96 & 0,98 & 0,95 & 1,00 \\
\hline PREC_A & 1,00 & - & - & - & 1,00 & - & - & - & 1,00 & - & - & - \\
\hline PREC_B & 0,84 & 1,00 & - & - & 0,95 & 1,00 & - & - & 0,95 & 1,00 & - & - \\
\hline PREC_C & 0,81 & 0,93 & 1,00 & - & 0,83 & 0,89 & 1,00 & & 0,81 & 0,89 & 1,00 & - \\
\hline PREC_D & 0,84 & 0,91 & 0,99 & 1,00 & 0,85 & 0,90 & 0,99 & 1,00 & 0,83 & 0,90 & 0,99 & 1,00 \\
\hline BLOQ_A & 1,00 & - & - & - & 1,00 & - & - & - & 1,00 & - & - & - \\
\hline BLOQ_B & 0,99 & 1,00 & - & - & 0,98 & 1,00 & - & - & 0,98 & 1,00 & - & - \\
\hline BLOQ_C & 0,95 & 0,93 & 1,00 & - & 0,94 & 0,95 & 1,00 & - & 0,94 & 0,95 & 1,00 & - \\
\hline BLOQ_D & 0,95 & 0,93 & 0,98 & 1,00 & 0,94 & 0,95 & 0,95 & 1,00 & 0,95 & 0,96 & 0,98 & 1,00 \\
\hline VM_A & 1,00 & - & - & - & 1,00 & - & - & - & 1,00 & - & - & - \\
\hline VM_B & 0,98 & 1,00 & - & - & 0,99 & 1,00 & - & - & 0,96 & 1,00 & - & - \\
\hline VM_C & 0,98 & 0,99 & 1,00 & - & 0,99 & 0,99 & 1,00 & - & 0,99 & 0,95 & 1,00 & - \\
\hline VM_D & 0,98 & 0,98 & 0,97 & 1,00 & 0,99 & 0,99 & 0,99 & 1,00 & 0,98 & 0,91 & 0,99 & 1,00 \\
\hline PASSE_A & 1,00 & - & - & - & 1,00 & - & - & - & 1,00 & - & - & \\
\hline PASSE_B & 0,97 & 1,00 & - & - & 0,93 & 1,00 & - & - & 0,94 & 1,00 & - & - \\
\hline PASSE_C & 0,91 & 0,82 & 1,00 & - & 0,93 & 0,78 & 1,00 & - & 0,94 & 0,81 & 1,00 & - \\
\hline PASSE_D & 0,88 & 0,76 & 0,99 & 1,00 & 0,94 & 0,80 & 0,99 & 1,00 & 0,95 & 0,82 & 0,99 & 1,00 \\
\hline
\end{tabular}

TABELA 4 - Resultados descritivos dos testes para os atletas de classificação baixa $(<2,0, n=6)$ e classificação alta $(>2,0, n=5)$.

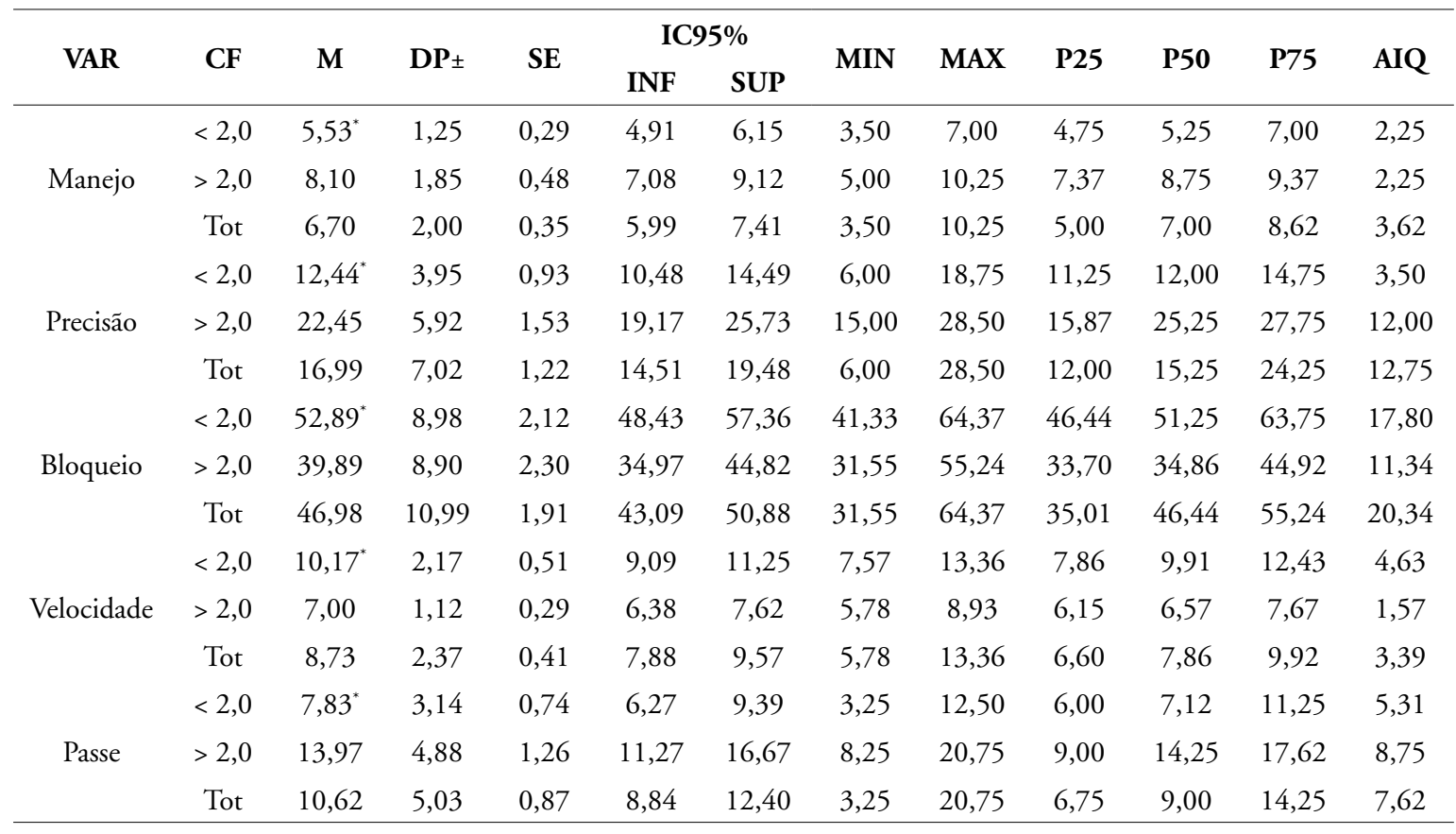




\section{Discussão}

As variáveis avaliadas pela bateria "Beck" são variáveis caracterizadoras do desempenho motor no "Rugby" em cadeira de rodas: Manejo de bola, Precisão de passes e passes de longa distância, bloqueios e deslocamento em velocidade. Portanto o objetivo do presente estudo, de analisar os critérios de autenticidade científica da bateria para torná-la válida como instrumento de avaliação, é relevante por prover meios consistentes de avaliação para a população Brasileira e, segue uma tendência de estudos com modalidades paraolímpicas que é observada na literatura.

Por exemplo, RAMOs e BARROS (2004) realizaram um estudo de revisão sobre avaliação motora adaptada cujo objetivo era de identificar os diferentes tipos de testes aplicáveis no basquetebol em cadeira de rodas. Na mesma modalidade Gorla, Araújo e CARMINATO (2005) analisaram as variáveis motoras de agilidade e força. KilKens, Post, Dallmeijer, Seelem e Van Der Woulde (2003) realizaram um extenso estudo de revisão sobre avaliação para sujeitos em cadeira de rodas, encontrando testes de habilidades de campo. Os autores concluíram que entre os 34 estudos identificados na literatura, havia uma grande variedade de tarefas e parâmetros de medida, e isto segundo os mesmos dificulta o processo de comparação dos resultados.

Brasile (1986, 1990) é um autor de trabalhos clássicos de avaliação no basquete em cadeira de rodas, em que questionava o sistema de classificação da Associação Americana de Basquete em Cadeira de Rodas (NWBA), utilizando o resultado obtido nos testes para correlacionar com a classe de cada atleta. No primeiro estudo o autor não encontrou diferenças significativas entre os resultados dos testes motores de grupos de atletas de duas classes distintas, o que forneceu base para questionar o sistema médico de classificação que levava apenas em consideração o nível neurológico da deficiência para classificar os atletas. Através do resultado da pesquisa, Brasile apresentou indicativos de que através do teste atletas de duas categorias estavam apresentando resultados similares, o que seria indício de falta de validade do sistema médico de classificação.

Doyle, Humphries, Dugan, Horn, Shim e NEWTON (2004) aplicaram um teste de velocidade de 20 metros para avaliar o sistema de classificação médico da NWBA, e comprovaram que não existiam diferenças significativas entre as classes II e III, o que sugere que o sistema de classificação funcional é mais eficaz para promover a igualdade entre os participantes.
Os estudos anteriormente citados ilustram a tendência encontrada na literatura de desenvolvimento e validação de instrumentos específicos para a avaliação de modalidades paraolímpicas. O presente estudo segue a esta tendência e através dos resultados aqui encontrados, considera-se que os testes satisfazem os critérios de autenticidade científica e, portanto são válidos para aplicação.

$\mathrm{O}$ procedimento de análise dos dados realizado foi semelhante ao utilizado por GoRGATTI e BÖHME (2003). Em seu estudo sobre um teste de agilidade modificada (zig-zag) para atletas de basquetebol em cadeira de rodas, as autoras analisaram o critério de objetividade através da ANOVA e com o coeficiente de correlação intraclasse analisaram a consistência interna.

A análise de variância de um fator (ANOVA one-way) utilizada neste estudo não apontou diferenças significativas entre os resultados de diferentes avaliadores, o que pode ser observado nas TABELAS 1 e 2. Este resultado é consistente com os resultados dos autores da bateria, Yilla e SHERriLL (1998) que através da mesma técnica, porém com medidas pareadas não encontraram diferenças entre os resultados dos diferentes avaliadores.

Quanto à análise da fidedignidade, os valores muito fortes de correlação linear permitem considerar que a bateria seja fidedigna, embora tenham sido encontradas diferenças estatisticamente significativas para alguns testes. $\mathrm{O}$ mesmo acontece em relação à consistência interna, pois foram encontrados fortes valores de correlação intraclasse com uma pequena exceção para a variável passe. Embora dois valores de correlação estejam abaixo da faixa considerada como muito boa (Avaliador 1, PASSE_B x PASSE_D, R = 0,76; Avaliador 2, PASSE_B x PASSE_C, $\mathrm{R}=0,78$ ), tais valores não são comprometedores de forma que acredita-se que a bateria seja consistente. Estes resultados estão em conformidade com os resultados de Yilla e SHERRILl (1998) que encontraram valor de F de 1,60 a 1,64 para os componentes da bateria, utilizando análise de variância para dados pareados, o que comprova a fidedignidade dos componentes da bateria.

Os testes que apresentaram valores de $\mathrm{r}$ inferiores a 0,90 foram, principalmente, os testes de precisão de passes de longa distância. Tais testes têm seus escores formados pela soma dos resultados de três arremessos em cada tentativa e, portanto uma pequena falha na execução dos arremessos pode ter influência direta sobre os resultados, o que pode justificar os valores abaixo de 0,90 para r. Analisando-se a TABELA 4 
percebe-se que para o avaliador $1 \mathrm{o}$ par A x B da variável manejo de bola apresentou uma correlação de 0,88 ( $p \leq 0,05)$, o que é um fator isolado visto que nenhum dos demais avaliadores apresentou valores semelhantes. As demais correlaçôes inferiores a 0,90 foram encontradas entre tentativas aplicadas em dias diferentes e isto pode ter sido causado por conta de outras variáveis (não controladas) que podem interferir no desempenho dos atletas na execução dos testes. Tratando-se de atletas com tetraplegia, esforços de vida diária, como por exemplo, deslocamentos de casa ao local de treinamento, podem demandar um desgaste energético que venha a influenciar a prática de atividade física e assim alterar diretamente o resultado do teste.

Os demais testes que são quantificados em pontos (manejo de bola) e segundos (bloqueio e velocidade) apresentaram valores acima de 0,90 , o que afasta a possibilidade de erro intra-avaliador ou erro inerente ao material utilizado (cronômetro, cadeira de rodas, demarcação do percurso entre outros).

Com relação aos valores referenciais divididos por classe funcional, ficaram evidentes diferenças significativas entre as classes, o que era esperado pela diferença de potencial funcional entre os jogadores (TABELA 4). Contudo, os valores do presente estudo são similares aos resultados apresentados no estudo original (Yilla \& Sherrill, 1998) cuja amostra era composta por 65 atletas americanos (este país possui uma das ligas mais tradicionais da modalidade). Para o teste de manejo de bola a média total do presente estudo foi de 6,7 em comparação com 9,66 do estudo de referência, sendo que o grupo de pontuação alta obteve uma média de 8,10 que se aproxima da referência. Para o teste de precisão de passes a média observada foi de 16,99 enquanto a referência é de 22,2 (média 22,45 para os atletas ponto alto deste estudo). O teste de bloqueio apresentou média de 46,98 segundos que pode ser considerado um tempo superior ao do estudo de referência, 63,98 segundos. Em relação ao teste de velocidade os atletas deste estudo apresentaram uma média superior referência 8,73 segundos contra 9,22. Finalmente para o teste de passes de longa distância a média foi inferior a referência 10,62 contra 15,18 (mesmo para o grupo de pontuação alta: 13,97).

A modalidade "Rugby" em cadeira de rodas é recente no Brasil. A realização deste estudo é a primeira iniciativa nacional de implementação de recursos para avaliação de atletas na modalidade. Estudos com esta população têm limitaçôes decorrentes da dificuldade com o contato com a amostra. Para delineamentos como o caso do que foi utilizado neste estudo, é preciso que os atletas tenham uma assiduidade aos treinamentos e isto limita a inclusão de atletas nas amostras. Outro fator limitante é ao fato de as equipes serem iniciantes e, grande parcela dos praticantes não ter a experiência necessária para atender os critérios de inclusão no estudo. Diante destes fatores ressalta-se a relevância deste estudo para o desenvolvimento da modalidade no país. Conclui-se desta pesquisa que a bateria é objetiva, fidedigna e consistente e pode ser utilizada na avaliação de atletas de "rugby" em cadeira de rodas com características semelhantes às dos atletas participantes da amostra.

\section{Abstract}

\section{Validation of beck battery of skills tests to Brazilian players of wheelchair rugby}

The Wheelchair Rugby is a sport practiced by subjects with tetraplegia or tetra-equivalence, since 2005 in Brazil, where there are approximately 10 teams in business. The motricity evaluation is a means of training control that allows achieve real progress in planning. The BECK battery of skills tests (ball handling, precision passing, blocking, speed and long distance passing) to wheelchair rugby athletes was developed in the U.S.A. by YILLA \& SHERRILL (1998).The aim of this study was to examine the criteria of scientific authenticity of the battery to the Brazilian athletes population. The results of two assessments with the battery (test and retest) with 11 athletes (mean age $27.59 \pm 5.37$ years, length of lesion 8.19 \pm 3.83 years), were recorded by three assessors, which allowed the analysis of objectivity, reliability and internal consistency. We used ANOVA for analysis of objectivity and linear correlation and test for analysis of reliability and consistency. No differences were found between results from different raters ( $p$ values ranging from 0.45 to 1.00 ) and the values of intraclass correlation coefficient found (range $r .78$ to $0.99 p<0.00)$ indicate that the battery is consistent and reliable. The results of this study indicate 
that the criteria of scientific authenticity to the battery are satisfied and battery can be considered a valid tool for motricity evaluation of wheelchair rugby brazilian players.

UNITERMS: Evaluation; Wheelchair sports; Physical disability.

\section{Notas}

1. O sistema de classificação funcional é uma forma de classificação esportiva através do qual se permite uma participação igualitária dentro das equipes. A variação é de 0,5 a 3,5 pontos com escala de 0,5 , sendo o jogador 0,5 o mais limitado funcionalmente e o 3,5 o menos limitado e, a soma dos quatro atletas em quadra não pode ultrapassar 8,0 pontos.

2. Os autores Anselmo de Athayde Costa e Silva e Leonarto Trevizan Costa são bolsistas do CNPq.

\section{Referências}

ASSOCIAÇÃO BRASILEIRA DE RUGBY EM CADEIRA DE RODAS. Informaçóes gerais sobre a modalidade. Rio de Janeiro: ABRC, 2009. Disponível em: <http://www.rugbiabrc.org.br/filiados.html>. Acesso em: 3 mar. 2009.

BRASILE, F. Performance evaluation of wheelchair athletes: more than a disability classification level issue. Adapted Physical Activity Quarterly, Champaign, v.7, p.289-97, 1990.

Wheelchair basketball skills proficiencies versus NWBA classifications. Adapted Physical Activity Quarterly, Champaign, v.3, p.6-13, 1986.

DOYLE, T.L.A.; HUMPHRIES, B.; DUGAN, E.L.; HORN, B.G.; SHIM, J.K.; NEWTON, R.U. Further evidence to change the Medical Classification System of the National Wheelchair Basketball Association. Adapted Physical Activity Quarterly, Champaign, v.21, p. 63-70, 2004.

GORGATTI, M.G.; BÖHME, M.T.S. Autenticidade científica de um teste de agilidade para indivíduos em cadeira de rodas. Revista Paulista de Educação Física, São Paulo, v.17, n.1, p.41-50, 2003.

GORLA, J.I.; ARAÚJO, P.F.; CARMINATO, R.A. Análise das variáveis motoras em atletas de basquetebol em cadeiras de rodas. Lecturas: Educación física y Deportes, Buenos Aires, v.1, n.83, 2005.

KILKENS, O.J.E.; POST, M.W.M.; DALLMEIJER, A.J.; SEELEM, H.A.M.; VAN DER WOULDE, L.H.V. Wheelchair Skills tests: a systematic review. Clinical Rehabilitation, London, v.17, p.418-30, 2003.

MOJTAHEDI, M.C.; VALENTINE, R.J.; EVANS, E.M. Body composition assessment in athletes with spinal cord injury: comparison of field methods with dual-energy X-ray absorptiometry. Spinal Cord, Houndmillls, v.47, p.698-704, 2009. RAMOS, G.C.; BARROS, J.F. Os diferentes tipos de avaliação física no basquetebol em cadeira de rodas e suas implicações para o crescimento deste esporte. Lecturas: Educación física y Deportes, Buenos Aires, v.1, n.72, 2004.

SANT'ANA, M.S. A história do rugby em cadeira de rodas no Brasil. In: GORLA, J.I.; CAMPANA, M.B. O rugby em Cadeira de Rodas: aspectos técnicos, táticos e diretrizes para o seu desenvolvimento e treinamento. [s.n.t], 2011. (no prelo). THOMAS, J.R.; NELSON, J.K.; SILVERMAN, S.J. Métodos de pesquisa em atividade física. 5. ed. Porto Alegre: Artmed, 2007.

TRITSCHLER, K.A. Barrow \& McGee’s pratical measurement and assessment. 5th. ed. Baltimore: Lippincott Williams \& Wilkins, 2000.

YILLA, A.B.; SHERRIL, C. Validating the Beck battery of quad rugby skills tests. Adapted Physical Activity Quarterly, Champaign, v.15, n.2, p.155-67, 1998. 
GORLA, J.I. et al.

ANEXO I - Bateria "Beck" de habilidades para "rugby" em cadeira de rodas n = 5.

\section{1) TESTE DE MANEJO DE BOLA}

Neste teste o atleta deve percorrer um percurso delimitado por cones durante o período de 30 segundos conduzindo a bola de acordo com as regras da modalidade, ou seja, a cada 10 segundos deve ser executado um drible e, no restante do tempo a bola pode estar no colo. O percurso é demarcado em um espaço de $10 \mathrm{~m}^{2}$. Os cones do percurso forma "portas" e, ao dar a volta em cada porta o atleta marca um ponto. O número de pontos marcados ao final do teste é o resultado. O teste é composto por duas tentativas válidas e a melhor pontuação é utilizada para a avaliação.

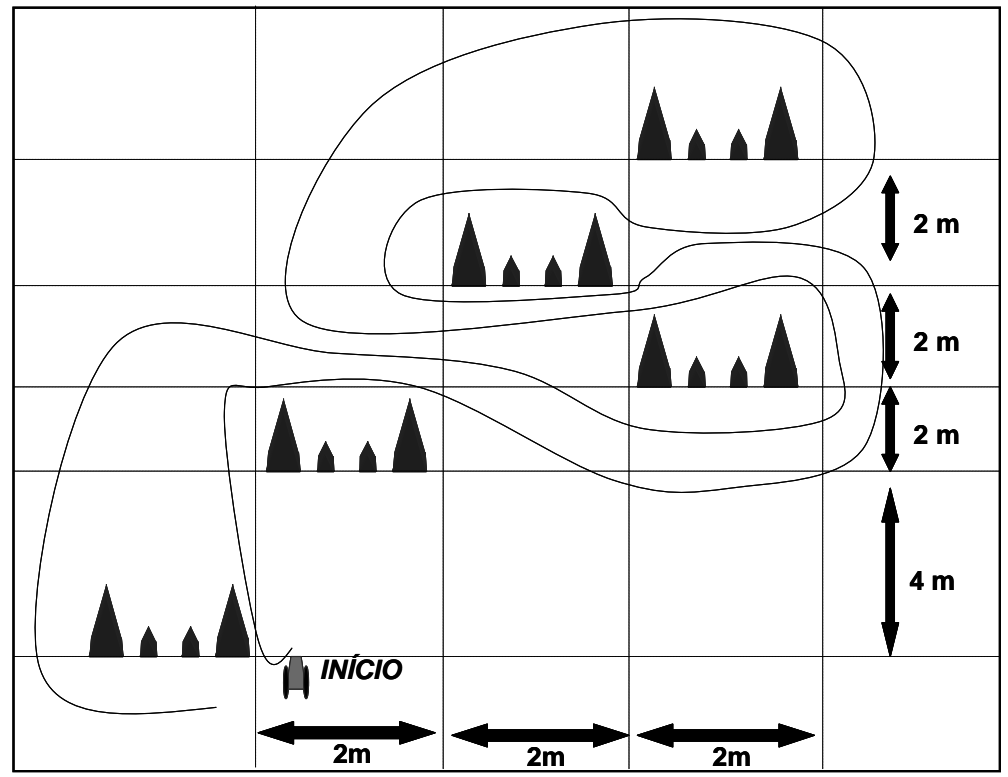

FIGURA 2 - Percurso do teste de manejo de bola.

\section{2) TESTE DE PRECISÃO DE PASSES}

Neste teste é desenhado um alvo na parede, cuja distância do sujeito avaliado é de cinco metros. A cada metro do sujeito à parede é passada uma linha, separando espaços cujos valores vão de um ponto na primeira parte à frente do avaliado ao centro do alvo que é de 10 pontos. A altura do alvo em relação ao chão é de $1,15 \mathrm{~m}$. O teste consiste em realizar passes até o alvo para quantificar a pontuação e o passe que o atleta utiliza em quadra deve ser utilizado no teste. Em cada tentativa do teste (duas ao todo) são três execuçōes. A soma dos pontos obtidos em cada tentativa é o resultado do teste. A FIGURA 3 ilustra a formação do alvo:

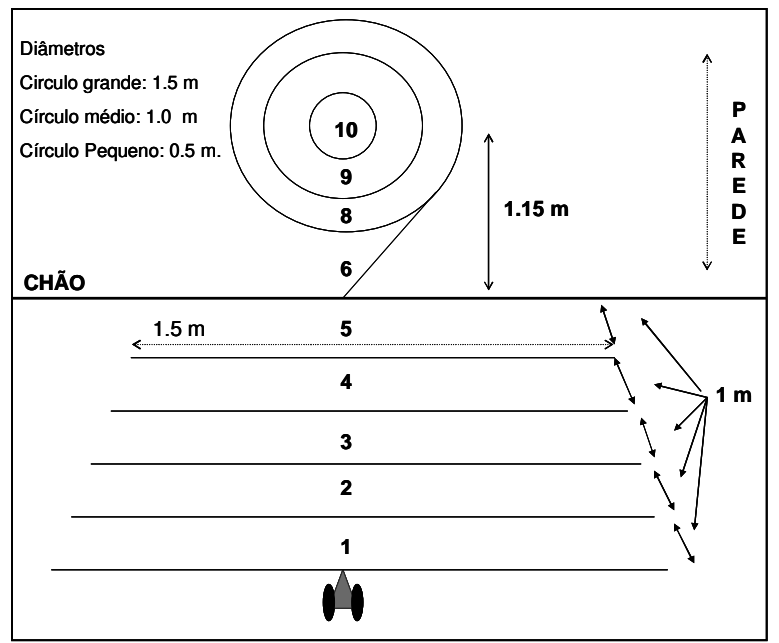

FIGURA 3 - Teste de precisão de passes.

484 • Rev. bras. Educ. Fís. Esporte, São Paulo, v.25, n.3, p.473-86, jul./ set. 2011 


\section{3) TESTE DE DESEMPENHO DE BLOQUEIO}

Este teste mensura a habilidade de bloqueio que é determinante para o desempenho do atleta nas partidas de "rugby" em cadeira de rodas. O teste consiste em um percurso de aproximadamente 24 metros, com seis cones colocados em linha reta, e com a distância de três metros entre cada um deles, e mais uma distância de 1,5 entre o início do percurso e o primeiro cone, bem como do último cone e término do percurso. Levando em consideração que a base dos cones pode variar de tamanho estimase em 24 metros a área de execução. Contudo para o presente estudo a distância foi de 22,8 metros por conta da largura dos cones. $\mathrm{O}$ atleta deve simular a ação do bloqueio em cada um dos cones. O resultado é o tempo em segundos da ação dos atletas.

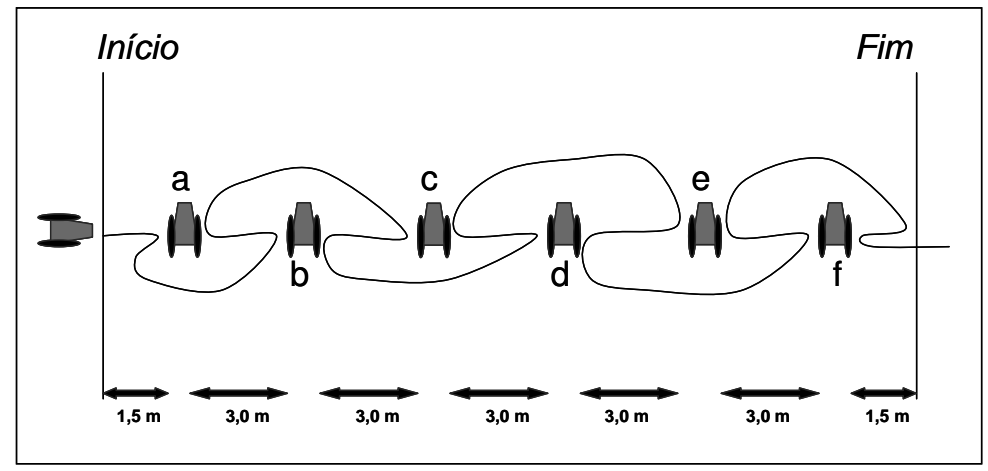

FIGURA 4 - Percurso do teste de desempenho de bloqueio.

\section{4) TESTE DE VELOCIDADE 20 METROS}

Este teste consiste em mensurar a habilidade de deslocamento em velocidade. Utiliza-se um percurso de 20 metros. O avaliador posiciona-se ao final do percurso e um auxiliar fica posicionado ao início para dar o sinal que corresponde à saída do atleta. O ponto de referência para o teste é a passagem do par de rodas dianteiras da cadeira, logo, quando o par de rodas dianteiras passa a linha o auxiliar que está com o braço elevado, abaixa-o para sinalizar ao avaliador que inicia a contagem do tempo com o cronômetro. O atleta desloca-se então em velocidade e quando passa pela linha final, o segundo avaliador para o cronômetro. O tempo em segundos é computado e são duas tentativas válidas no teste.

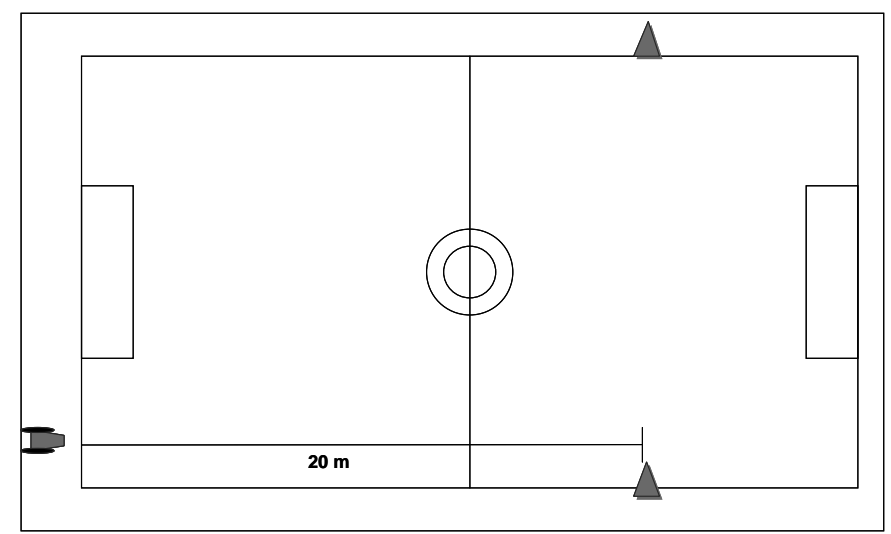

FIGURA 5 - Percurso do teste velocidade 20 metros. 
GORLA, J.I. et al.

\section{5) TESTE DE PASSES DE LONGA DISTÂNCIA}

Este é o teste que visa mensurar a habilidade dos atletas em efetuar os passes de longa distância. O participante posicionase à linha de fundo da quadra e, deve realizar o passe de longa distância com a maior força possível, utilizando o passe realizado durante a situação de jogo. Lateralmente à quadra são demarcados espaços de dois em dois metros com cones que são as zonas de referência para a pontuação do teste. As zonas são enumeradas com escala de razão (1) a partir do número um. A pontuação da zona em que a bola toca ao chão é o resultado da execução. São três execuções em cada uma das duas tentativas. A soma em cada tentativa é o resultado do teste.

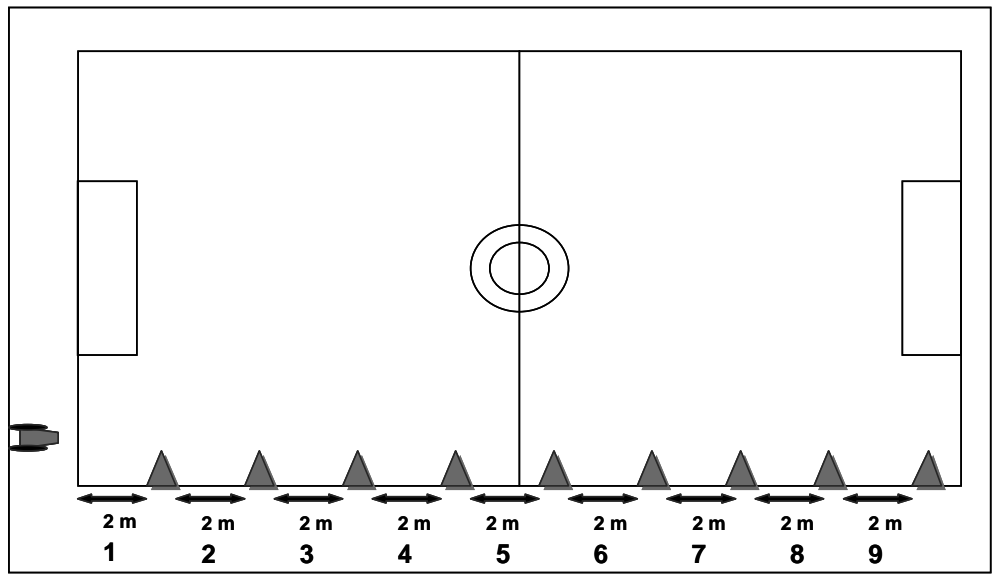

FIGURA 6 - Teste de passes de longa distância.

\section{Agradecimentos}

Este estudo foi financiado pela Fundação de Amparo à Pesquisa do Estado de São Paulo, através do edital de apoio à pesquisa permanente.

\begin{tabular}{r|l} 
ENDEREÇO & \\
J osé Irineu Gorla & \\
R. General Osório, 39 - apto. 123 & Recebido para publicação: 30/06/2010 \\
13271-130 - Valinhos - SP - BRASIL & Revisado: 04/05/2011 \\
e-mail: jigorla@uol.com & Aceito: 30/05/2011 \\
&
\end{tabular}

